\title{
A Novel Polyelectrolyte Complex (PEC) Hydrogel for Controlled Drug Delivery to the Distal Intestine
}

\author{
C. Zaino ${ }^{1}$, Y. Zambito ${ }^{1}$, G. Mollica ${ }^{2}$, M. Geppi ${ }^{2}$, M. F. Serafini ${ }^{1}$, V. Carelli ${ }^{1}$ and G. Di Colo ${ }^{*}, 1$ \\ ${ }^{1}$ Department of Bioorganic Chemistry \& Biopharmaceutics, University of Pisa, Via Bonanno 33, 56126 Pisa, Italy \\ ${ }^{2}$ Department of Chemistry \& Industrial Chemistry, University of Pisa, Via Risorgimento 35, 56126 Pisa, Italy
}

\begin{abstract}
This work was aimed at preparing and evaluating a physically crosslinked hydrogel for the controlled release of diverse drugs to the distal intestine. A solution of fluorescein isothiocyanate dextran, MW $4400 \mathrm{Da}$ (FD4), or a dispersion of micronized dexamethasone (DMS) was microencapsulated into a PEC hydrogel, composed of polycationic N-trimethyl chitosan (TMC) and polyanionic N-carboxymethyl chitosan (CMCh). A fine spray of a $1 \% \mathrm{CMCh}$ solution containing $1 \%$ FD4 in solution or $0.1 \%$ DMS in dispersion was directed into a $2 \%$ TMC solution, then the resulting microcapsules (MCPS) were lyophilized. MCPS were analyzed by SEM and solid-state NMR. Drug release from MCPS was too fast, so these were compressed into matrices (weight $20 \mathrm{mg}$; diameter $6 \mathrm{~mm}$; drug load 2.5\%, with FD4, or 3.7\%, with DMS) which were enteric coated. Drug release from matrices was studied simulating matrix transit across GI environments of different $\mathrm{pHs}$, from stomach to proximal colon. The enteric film hindered release in stomach and proximal small intestine. After film dissolution at ileum $\mathrm{pH}$, release occurred with a pattern described by the Peppas equation ( $\mathrm{n}=0.6$, with DMS; $\mathrm{n}=0.7$, with FD4). As the $\mathrm{pH}$ changed from 7.4 to 6 (from ileum to ascending colon) MCPS were liberated from matrix surface. This phenomenon sustained the release rate. The present MCPS allow controlled doses of macromolecular or microparticulate drugs being uniformly loaded into controlled-release matrices based on a physically crosslinked, biodegradable hydrogel.
\end{abstract}

\section{INTRODUCTION}

Hydrogels are defined as macromolecular networks swollen in water or biological fluids [1]. The considerable amount of water they are able to absorb makes them similar to living tissues, therefore, they are biocompatible and suitable for biomedical and pharmaceutical applications [2]. The networks of chemical hydrogels are formed by irreversible, covalent crosslinking. However, free unreacted covalent crosslinkers are potentially toxic, therefore alternative types of networks, formed by direct physical interactions between polymer chains without the addition of crosslinkers, have been developed [3]. A component of these hydrogel types is chitosan, a polycationic biocompatible polymer generally produced at low cost by alkaline deacetylation of chitin, which is the main component of the exoskeleton of crustaceans [4]. These hydrogels were formed by chitosan complexation with an oppositely charged polyelectrolyte.

The electrostatic attraction between the positively charged amino groups of chitosan and the negatively charged groups of the other polyelectrolyte led to the formation of the polyelectrolyte complex (PEC). PECs have been used to prepare drug delivery systems. Their essential properties have been reviewed by Berger et al. [3]. In order to form a PEC both component polyelectrolytes must be in the ionized form. Chitosan is in the unionized amine form at the neutralalkaline $\mathrm{pH}$ of physiological application sites of many drug delivery systems, while the anionic polyelectrolyte is in the ionized form. In these conditions no stable PEC can be

*Address correspondence to this author at the Department of Bioorganic Chemistry \& Biopharmaceutics, University of Pisa, Pisa, Italy; Tel+39-0502219656; Fax: +39-050-2219660; E-mail: giadic@ farm.unipi.it formed. Therefore it could be convenient to use, as the polycation, the quaternized chitosan derivative N-trimethyl chitosan (TMC), which is ionized irrespective of $\mathrm{pH}$. Although TMC is not commercially available as yet, vast literature information is available on its synthesis from chitosan, purification, biopharmaceutical properties, safety (see e.g., Ref. 5). In fact, TMC is considered as safe, biocompatible and biodegradable as the parent chitosan. A biocompatible biodegradable and safe polyanion could be obtained by grafting carboxymethyl groups onto the chitosan backbone, to yield $\mathrm{N}$-carboxymethyl chitosan (CMCh) [6].

In the present work microcapsules (MCPS) entrapping controlled amounts of fluorescein isothiocyanate dextran, MW 4400 Da (FD4), a model of water-soluble macromolecular drugs, or of fine particles of the barely soluble antiinflammatory dexamethasone (DMS), into a PEC hydrogel at physiological $\mathrm{pH}$ have been prepared by directing a fine spray of a CMCh solution containing dissolved FD4 or dispersed DMS into a TMC solution. The mechanism of MCPS formation, the encapsulation efficiency and the in vitro drug release from MCPS to simulated intestinal environment have been investigated. PEC MCPS have been compressed into monolithic matrices, these have been enteric coated with Eudragit S100, and drug release from the coated systems to simulated GI fluids has been studied in vitro. The aim has been the design of a biocompatible and biodegradable system for the controlled and complete release of either macromolecular drugs, such as peptides and proteins, or barely water-soluble drugs, such as DMS, to the distal intestine. After dissolution of the enteric coating and swelling of the hydrogel, the release of the water-soluble macromolecular FD4 is expected to be comparatively slow due to its intrinsically low diffusion coefficient in the hydrogel, while the poor solubility of DMS should be 
responsible for a slow dissolution of this drug from the hydrogel. On the other hand, the high solubility of FD4 and the high specific surface area and diffusivity of DMS in the aqueous phase of the hydrogel should allow the release of these drug models from the hydrogel being complete during transit across the intestinal tract.

\section{MATERIALS AND METHODS}

\section{Materials}

Dexamethasone (DMS), fluorescein isothiocyanate dextran, MW $4400 \mathrm{Da}$ (FD4), chitosan from crab shells (ChC, minimum $85 \%$ deacetylated, viscometric MW $900 \mathrm{KDa}$ ) were purchased from Sigma. Chitosan from shrimps (ChS, minimum 90\% deacetylated, viscometric MW 590 KDa) (ChitoClear FG90, Primex, Drammen, Norway) was kindly gifted by Giusto Faravelli, Milan, Italy. Eudragit S100 and triethyl citrate were kindly gifted by Rofarma Italia, Milan, Italy. Ntrimethyl chitosan (TMC) was synthesized from ChS as described by Di Colo et al. [7]. N-carboxymethyl chitosan (CMCh) was synthesized from $\mathrm{ChC}$ as described by Di Colo et al. [8]. DMS (water solubility, $0.1 \mathrm{mg} / \mathrm{ml}$ [9]) was obtained as microparticles $(<1.3 \mu \mathrm{m})$ by spray-drying a $0.09 \mathrm{mg} / \mathrm{ml}$ solution (Mini Spray Drier BÜCHI B-191, inlet and outlet temperature, $150^{\circ} \mathrm{C}$ and $60^{\circ} \mathrm{C}$, respectively; spray nozzle, 0.7 $\mathrm{mm}$; feed flow, $8 \mathrm{ml} / \mathrm{min}$ ).

\section{Preparation and Characterization of PEC Microcapsules (MCPS)}

PEC MCPS containing FD4 in solution or DMS microparticles in dispersion were obtained by directing a spray of a $1 \%$ aqueous $\mathrm{CMCh}$ solution containing $1 \%$ FD4 or $0.1 \%$ DMS microparticles (two-fluid nozzle, diameter $0.4 \mathrm{~mm}$; air pressure, 1 bar; spray rate $2 \mathrm{ml} / \mathrm{min}$ ) into a $2 \%$ TMC solution. The volume of the TMC solution was double that of the CMCh solution. The MCPS were collected by centrifugation (MLW $\mathrm{T}$ 52.2; $3000 \mathrm{rpm}, 5 \mathrm{~min}$ ), repeatedly (two-three times) washed with water, and the sediment lyophilized (Virtis, Advantage-53; freezing step: $-35^{\circ} \mathrm{C}$ in $180 \mathrm{~min}$; drying cycle steps: 1) $\left.-30^{\circ} \mathrm{C}, 360 \mathrm{~min} ; 2\right)-10^{\circ} \mathrm{C}, 360 \mathrm{~min}$; 3) $10^{\circ} \mathrm{C}, 240$ min; 4) $25^{\circ} \mathrm{C}, 180 \mathrm{~min}$ ). Non-medicated PEC MCPS were also prepared. The output of each process of MCPS preparation was expressed as grams of lyophilized material obtained from $1 \mathrm{~g}$ of CMCh. The ability of the different processes to produce MCPS from plain CMCh was compared via the respective output values.

\section{MCPS Size}

Immediately after preparation of the MCPS dispersion, this was observed on an optical microscope equipped with a stage micrometer.

\section{MCPS Morphology}

The shape and surface morphology of lyophilized MCPS, either drug loaded or drug free, were examined by scanning electron microscopy (SEM) (Jeol JSM 5600LV) after coating with gold by sputter-coating (Edwards S150B).

\section{Solid State NMR Measurements}

All the ${ }^{1} \mathrm{H}$ and ${ }^{13} \mathrm{C}$ high-resolution solid-state NMR experiments were performed on $\mathrm{CMCh}, \mathrm{TMC}$ and lyophilized
MCPS using a Varian InfinityPlus 400 double channel spectrometer operating at the ${ }^{1} \mathrm{H}$ frequency of $399.89 \mathrm{MHz}$ and ${ }^{13} \mathrm{C}$ frequency of $100.56 \mathrm{MHz}$, equipped with a CP-MAS probe for rotors with an outer diameter of $3.2 \mathrm{~mm}$. Both ${ }^{13} \mathrm{C}$ and ${ }^{1} \mathrm{H} 90^{\circ}$ pulses were $2.1 \mu \mathrm{s}$. The carbon spectra were acquired under Magic Angle Spinning (MAS) and high-power decoupling conditions, using the Cross Polarization (CP) pulse sequence and a spinning rate of $6 \mathrm{kHz}$ : the optimized contact time and relaxation delay were $0.5 \mathrm{~ms}$ and $5 \mathrm{~s}$ for both CMCh and TMC, and $0.2 \mathrm{~ms}$ and $10 \mathrm{~s}$ for the lyophilized MCPS, respectively. The ${ }^{13} \mathrm{C}$ Direct Excitation (DE) spectra for all samples were acquired using a depth pulse sequence for background suppression [10] at the spinning rate of $6 \mathrm{kHz}$ with a relaxation delay of $2 \mathrm{~s}$.

${ }^{1} \mathrm{H}$ low-resolution solid-state NMR experiments were performed at the Larmor frequency of $25 \mathrm{MHz}$ on a singlechannel Varian XL-100 spectrometer interfaced with a DSNMR Stelar acquisition system and equipped with a $5 \mathrm{~mm}$ probehead. In all experiments the Free Induction Decays (FID) were acquired in on-resonance conditions following a solid-echo pulse sequence to avoid the dead time effect; the $90^{\circ}$ pulse length and the echo delay were 2.7 and $13 \mu \mathrm{s}$, respectively.

\section{Drug Release Kinetics, Drug Content, and Entrapment Efficiency}

Medicated MCPS (about $5 \mathrm{mg}$, accurately weighed) were added to a screw-capped vial containing $25 \mathrm{ml}$ of phosphate buffer $\mathrm{pH} 7.4,0.13 \mathrm{M}$. The vial was fixed radially to a rotating wheel $(26 \mathrm{rpm}) \mathrm{kept}$ in an atmosphere thermostated at $37^{\circ} \mathrm{C}$. Sink conditions were always ensured. At appropriate time intervals, 1-ml aliquots were withdrawn, centrifuged, and the surnatant was analyzed for the drug by HPLC. To determine the drug content in MCPS, these were completely depleted of drug. To this purpose the release experiments were protracted for $24 \mathrm{~h}$, then the dissolution medium was analysed for the drug, and the absence of any further drug release was verified. Depletion was generally complete within $24 \mathrm{~h}$. The entrapment efficiency was expressed as the ratio of the drug mass contained in the lyophilized MCPS to the total drug mass sprayed into the TMC solution. The latter was calculated knowing the drug concentration in the sprayed CMCh solution and the total volume of this solution.

\section{HPLC Analysis}

The HPLC techniques described by Sandri et al. [11] and by Zambito et al. [12] were used for the analysis of FD4 and DMS, respectively.

\section{FD4}

The apparatus (Perkin-Elmer) consisted of Series 200 pump, $20 \mu \mathrm{l}$ Rheodyne injector, Series 200 fluorescence detector and Turbochrom Navigator HPLC software for data integration. A C18 column Hyperbond C18 $10 \mu \mathrm{m}, 300$ $\mathrm{mm} \times 3.9 \mathrm{~mm}$, was used. The mobile phase consisted of 2.5 $\mathrm{mM} \mathrm{KH} \mathrm{PO}_{4} \mathrm{pH} 5.0$ (91.5\%) and acetonitrile (8.5\%). The flow rate was $1 \mathrm{ml} / \mathrm{min}$. The detection wavelengths were: excitation $\lambda=490 \mathrm{~nm}$ and emission $\lambda=515 \mathrm{~nm}$. A calibration curve was constructed using FD4 concentrations ranging from 0.5 to $20 \mu \mathrm{g} \mathrm{ml}^{-1}$. Linearity was observed in the above concentration range $\left(\mathrm{r}^{2}>0.99\right)$. 


\section{DMS}

The apparatus described above was used, with UV detection (Perkin Elmer LC 290) set at $241 \mathrm{~nm}$. A Spheri-5 RP18 $250 \mathrm{~mm} \times 4.6 \mathrm{~mm} 5 \mu \mathrm{m}$ column was used. The mobile phase (flow rate $1.0 \mathrm{ml} / \mathrm{min}$ ) was water-acetonitriletetrahydrofuran $(64: 35: 1)$. A calibration curve was constructed using DMS concentrations ranging from 0.5 to 20 $\mu \mathrm{g} \mathrm{ml}^{-1}$. Linearity was observed in the above concentration range $\left(r^{2}>0.99\right)$.

\section{Drug Release from Monolithic Matrices Prepared from PEC MCPS}

\section{Preparation of Matrices}

The lyophilized MCPS, either drug containing or drug free, were directly compressed into circular flat-faced monolithic matrices of $20 \mathrm{mg}$ weight and $6 \mathrm{~mm}$ diameter. For compaction, a force of $1000 \mathrm{~kg}$ was applied for $15 \mathrm{~s}$ by a hydraulic press (Perkin-Elmer).

\section{Swelling Kinetics of Matrices}

At time zero, the matrices were immersed in water at $37^{\circ} \mathrm{C}$. At appropriate intervals they were withdrawn and rapidly blotted dry and weighed $\left(10^{-5} \mathrm{~g}\right)$. The swelling degree was expressed as ratio of swollen to initial dry weights. The swelling kinetics were measured for $24 \mathrm{~h}$.

\section{Enteric Coating of Matrices}

Matrices were enteric coated by a spraying technique. The matrices were placed on a rotating plate $(37 \mathrm{rpm})$ and sprayed on the upper face and on the edge with a methanolic solution of 5\% Eudragit S100 and $0.5 \%$ triethyl citrate. The two-fluid spray nozzle (diameter, $0.4 \mathrm{~mm}$; air pressure, 1 bar; spray rate, $7.5 \mathrm{ml} / \mathrm{min}$ ) was kept in a fixed position, so that the spray wetted a limited portion of the rotating plate surface, centered at a $3.5-\mathrm{cm}$ distance from the rotation axis. The matrices were placed on the plate in circle, at this distance from the axis, so that each matrix was wetted by the spray for about $0.4 \mathrm{~s}$ for every rotation of the plate. Every 4th rotation, the spraying was interrupted, the coating was dried by an air stream at room temperature, the matrices were turned upside-down and an equal coating was applied on the other face. The process was stopped when the weight of the coating reached $5 \pm 0.2 \mathrm{mg}$.

\section{Composition of Simulated GI Fluids}

Simulated gastric fluid (SGF) consisted of $\mathrm{HCl} 0.04 \mathrm{M}$, $\mathrm{pH} 1.2$, made isotonic with $\mathrm{NaCl}$; simulated jejunal fluid (SJF) was phosphate buffer $\mathrm{pH} 6.8,0.13 \mathrm{M}$, made isotonic with $\mathrm{NaCl}$; simulated ileal fluid (SIF) was phosphate buffer $\mathrm{pH} 7.4,0.13 \mathrm{M}$, isotonic.

\section{Preparation of Simulated Ascending Colon Environment (SACE)}

\section{A) In the Absence of Cecal Contents}

A phosphate buffer $\mathrm{pH} 6.0$ containing $0.073 \mathrm{~g} / \mathrm{CaCl}_{2}$, $0.087 \mathrm{~g} / 1 \mathrm{MgCl}_{2}, 0.450 \mathrm{~g} / \mathrm{l} \mathrm{KCl}, 28.126 \mathrm{~g} / \mathrm{l} \mathrm{NaH} \mathrm{PO}_{4}$, and $4.992 \mathrm{~g} / 1 \mathrm{Na}_{2} \mathrm{HPO}_{4}$ (code, SACE/PB) was used.

For some experiments an acetate buffer $\mathrm{pH}$ 6.0, containing $14.96 \mathrm{~g} / 1 \mathrm{CH}_{3} \mathrm{COONa} 3 \mathrm{H}_{2} \mathrm{O}, 0.075 \mathrm{~g} / \mathrm{l} \mathrm{NaCl}, 340 \mu \mathrm{l} / 1$ glacial $\mathrm{CH}_{3} \mathrm{COOH}$ (code, SACE/AB) was used.

\section{B) In the Presence of Cecal Contents}

About $3 \mathrm{~g}$ of cecal contents, taken from a Wistar rat, were diluted 1:3 with SACE/PB, the dispersion was immediately centrifuged (3000 rpm, $10 \mathrm{~min})$ and the surnatant ultracentrifuged $\left(39000 \mathrm{rpm}, 15^{\circ} \mathrm{C}, 30 \mathrm{~min}\right)$. The surnatant from the ultracentrifugation was saturated with $\mathrm{CO}_{2}$ to attain a constant pH of 5.6 (code, SACE/PB/CC).

\section{Measurement of Release Kinetics}

The apparatus was the same as that described above for the release experiments with the MCPS. Each matrix, containing FD4 or DMS, was agitated at $37^{\circ} \mathrm{C}$ in $5 \mathrm{ml}$ (case of FD4) or $25 \mathrm{ml}$ (case of DMS) of dissolution medium, simulating the environment of a specific region of the GI tract, for a specified time period, after which the medium was replaced by a different one to simulate matrix transit across different GI regions. Sink conditions were always ensured. At appropriate intervals, $100-\mu 1$ (case of FD4) or 20-ml (case of DMS) samples of each dissolution medium were withdrawn and replaced with equal volumes of fresh pre-thermostated medium. A superficial matrix disintegration was observed during elution with the buffers having $\mathrm{pH} \leq 6$ (SACE/PB, SACE/AB, SACE/PB/CC). In these cases the vial was subjected to centrifugation $(3000 \mathrm{rpm}$, $2 \mathrm{~min}$ ) before sampling. Samples were analyzed for FD4 and DMS by the respective above-mentioned HPLC techniques, and the cumulative drug fraction released was calculated. The uncoated matrices were generally eluted with SIF for $2 \mathrm{~h}$, followed by SACE/PB, or SACE/AB, or SACE/ PB/CC, for the successive $4 \mathrm{~h}$. The enteric-coated matrices were eluted in sequence with SGF (2 h), SJF (2 h), SIF (2 h), and SACE/PB $(4 \mathrm{~h})$.

\section{Release Data Treatment}

The release data were analyzed, up to $50-60 \%$ release, by the following phenomenological equation, known as Peppas equation [13]:

$$
F=K t^{n}
$$

where $\mathrm{F}$ is the dose fraction released in time $\mathrm{t} ; \mathrm{K}$ is a rate parameter; and the time exponent, $\mathrm{n}$, measures the curvature of the release profile, hence, is related to the release mechanism. Eq. (1) is applicable up to a released fraction of $60 \%$.

\section{RESULTS AND DISCUSSION}

\section{Preparation and Characterization of PEC MCPS}

Optical microscopy was used to measure the size interval of suspended MCPS, before their collection.

Spherical particles in the size ranges listed in Table $\mathbf{1}$ were made out.

Table 1. Characteristics of the microencapsulation process. Means \pm SD $(n=8)$

\begin{tabular}{|c|c|c|c|c|}
\hline Drug & Output $^{\mathbf{a}}$ & MCPS size Range, $\boldsymbol{\mu} \mathbf{m}$ & DL $^{\mathbf{b}}, \boldsymbol{\%}$ & $\mathbf{E E}^{\mathbf{c}}, \boldsymbol{\%}$ \\
\hline \hline- & $0.838 \pm 0.002$ & $20-100$ & - & - \\
\hline FD4 & $0.840 \pm 0.016$ & $20-100$ & $2.5 \pm 0.1$ & $2.1 \pm 0.1$ \\
\hline DMS & $0.657 \pm 0.007$ & $20-100$ & $3.7 \pm 0.1$ & $24.3 \pm 2.5$ \\
\hline
\end{tabular}

${ }^{a}$ Grams of lyophilizate per gram of CMCh

${ }^{\mathrm{b}}$ Drug load in MCPS.

${ }^{c}$ Entrapment Efficiency. 
The drug-free ones and those prepared with a solution of FD4 appeared clear, whereas those prepared with a dispersion of DMS appeared opaque, indicating the entrapment of DMS microparticles (size, $<1.3 \mu \mathrm{m}$ ) within the microspheres (size, 20-100 $\mu \mathrm{m}$ ). This analysis, however, could not make clear the structure of microspheres, i. e., these could be either monolithic particles or MCPS. In order to clarify this point, a $\mathrm{CMCh}$ solution was dripped into a solution of an excess TMC: the formation of macroscopic capsules of the same size as the CMCh solution drops was observed, as shown in Fig. (1).

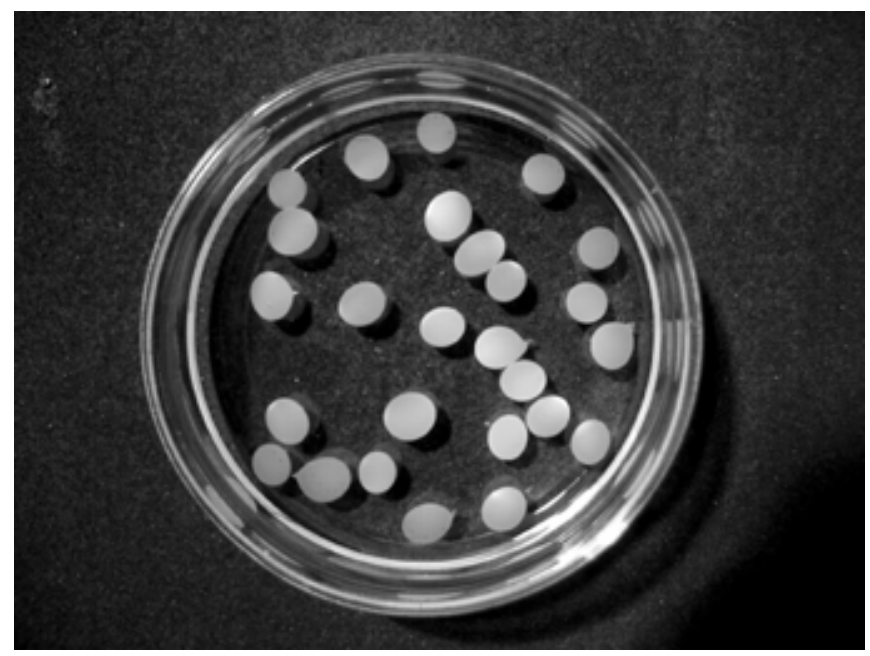

Fig. (1). Photograph of macroscopic capsules obtained by dripping a CMCh solution into a solution of an excess TMC.

It was also observed that when the opposite was done, namely, a TMC solution was dripped into a solution of $\mathrm{CMCh}$ in excess, small particles of irregular shape were formed instead of macroscopic capsules. Probably, in the former instance the formation of the CMCh-TMC complex at the interface between the drops of CMCh solution and the external TMC solution was faster than the dispersion of the drops into the solution bulk. The PEC would form a spherical wall at the interface, which would prevent further mixing of polymers. The dispersion of the drops was comparatively slow because of the comparatively high viscosity of the CMCh solution. On the other hand, when the TMC solution, having a comparatively low viscosity, was dripped into the $\mathrm{CMCh}$ solution, the drops would disperse more rapidly, therefore small and irregular PEC particles were formed. These considerations are believed to apply as well to the microdrops of CMCh solution sprayed into the TMC solution, hence the microparticles, the properties of which are listed in Table 1, should in fact be MCPS entrapping a solution of FD4 or a dispersion of DMS. The low SD values appearing in Table 1 testify to a good reproducibility of the preparation procedure. The output of such a procedure for the drug-free and the FD4 MCPS was virtually equal, whereas it was significantly lower for the DMS MCPS. Apparently, the conversion of the microdrops of the DMS dispersion into MCPS was comparatively difficult. Indeed, for the PEC wall to be formed at the microdrop surface $\mathrm{CMCh}$ had to diffuse from the inside of the drop to the drop surface. Such a diffusion could well be hindered by the DMS microparticles dispersed in the microdrops, so less TMC was adsorbed on the drop surface in the case of DMS than in that of FD4 or of the drug-free microdrops. On the other hand, the entrapment efficiency (EE) was much higher with the DMS dispersion than with the FD4 solution. In fact, in the latter case the EE value seen in Table $\mathbf{1}$ is exceedingly low, which is indicative of a pronounced tendency of the FD4 molecules to escape from MCPS during their formation. With DMS the EE value is much higher, possibly because the solution outside the MCPS soon became saturated with this barely soluble drug, thus limiting its diffusion-driven leakage.

The SEM micrograph in Fig. (2) shows the collapsed PEC shell of a DMS MCPS from the inside of which water has been removed by lyophilization. None of the DMS microparticles that were seen by optical microscopy to be associated with hydrated MCPS appear on the smooth PEC surface, thus confirming the entrapment of such particles within the PEC MCPS. SEM micrographs of lyophilized FD4 or drug-free MCPS are not shown, as quite similar to that in Fig. (2). The high-resolution solid-state NMR ${ }^{13} \mathrm{C}$ spectra of CMCh, TMC and lyophilized MCPS are reported in Fig. (3), where an expansion of the spectral region of interest (40-120 $\mathrm{ppm}$ ) is shown. The quite broad linewidth observed for all samples indicates a very low degree of structural order, already observed in chitosans in opposition to the high crystallinity of $\alpha$-chitin [14-16]. Nevertheless, all signals in the TMC spectrum generally appear narrower than the corresponding signals for $\mathrm{CMCh}$, because of the presence of faster reorientational motions. This is in agreement with the lack of hydrogen bonds in non-acetylated units, due to the presence of trimethylammonium groups [17]. The signals in the ${ }^{13} \mathrm{C}$ spectra of the two polyions could be assigned by comparison with literature data and by semiempirical ${ }^{13} \mathrm{C}$ chemical shift calculations as reported in Fig. (3).

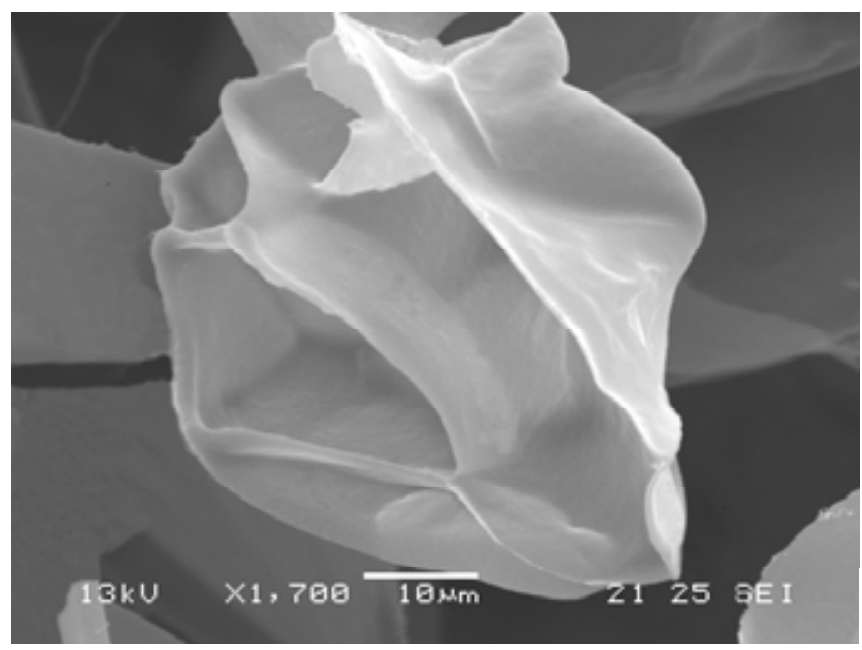

Fig. (2). SEM micrograph showing a lyophilized microcapsule containing DMS.

In particular, the side-group carbon signals for both $\mathrm{CMCh}$ and TMC have been identified by acquiring selective ${ }^{13} \mathrm{C}$ DE-MAS spectra, where the use of a $2 \mathrm{~s}$ relaxation delay allows signals arising from nuclei experiencing high mobility to be relatively enhanced $[18,19]$. In particular, the signal at $55.6 \mathrm{ppm}$ in the TMC spectrum was assigned to the methyl carbons of the trimethylammonium group, which are expected to undergo fast rotations, also corresponding to a 


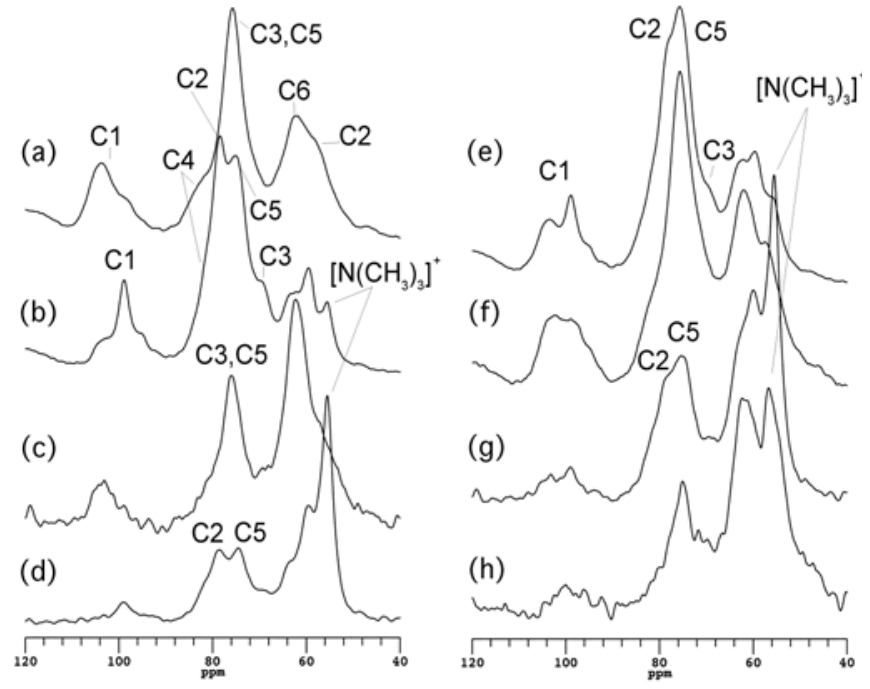

Fig. (3). Expansions of the high-resolution solid-state NMR 13C CP-MAS and DE-MAS spectra of CMCh and TMC (left), and lyophilized MCPS (right). (a), (b), (f) and (e): 13C CP-MAS spectra of CMCh, TMC, MCPS (experimental), and MCPS (calculated), respectively. (c), (d), (h) and (g): 13C DE-MAS spectra of CMCh, TMC, MCPS (experimental), and MCPS (calculated), respectively. CP spectra were acquired at a spinning speed of $6 \mathrm{kHz}$, using a contact time and a relaxation delay of $0.5 \mathrm{~ms}$ and $5 \mathrm{~s}$ for both CMCh and TMC, and $0.2 \mathrm{~ms}$ and $10 \mathrm{~s}$ for the MCPS sample, respectively. DE spectra were acquired at a spinning speed of $6 \mathrm{kHz}$, using a relaxation delay of $2 \mathrm{~s}$. The MCPS calculated spectra were obtained by mathematically adding up the corresponding spectra of CMCh and TMC.

very narrow signal at $\approx 3.5 \mathrm{ppm}$ in the ${ }^{1} \mathrm{H}$ MAS spectrum of TMC (not shown). The ${ }^{13} \mathrm{C}$ CP- and DE-MAS spectra of lyophilized MCPS are reported in Fig. (3f,h), respectively, together with those obtained by mathematically adding up either the CP- or the DE-MAS spectra of CMCh and TMC (Fig. (3e,g), respectively). The calculated spectra have the scope of highlighting possible dynamic or conformational changes occurring in the MCPS with respect to the pure CMCh and TMC components. Compared to the calculated spectrum, the experimental ${ }^{13} \mathrm{C}$ CP-MAS spectrum of MCPS shows a significant broadening of the two signals at $\approx 99.2$ and $\approx 55 \mathrm{ppm}$, possibly due to a reduction in the mobility of the TMC units with respect to the pure sample, and the displacement of the signal at $78.8 \mathrm{ppm}$, due to interactions with $\mathrm{CMCh}$ involving the $\mathrm{C} 2$ carbon of TMC. These observations are supported by the comparison between the experimental and calculated ${ }^{13} \mathrm{C}$ DE-MAS spectra. In fact, the signal at $\approx 55 \mathrm{ppm}$ is less intense in the experimental than in the calculated spectrum, clearly indicating a reduction of mobility for the $\mathrm{CH}_{3}$ trimethylammonium groups in MCPS, confirmed by the remarkable broadening of the peak at $\approx 3.5 \mathrm{ppm}$ in the corresponding ${ }^{1} \mathrm{H}$ MAS spectrum.

In order to confirm these results and obtain more detailed and quantitative information on the molecular dynamics of these systems, an analysis of the ${ }^{1} \mathrm{H}$ Free Induction Decay (FID) of CMCh, TMC, and MCPS samples acquired in lowresolution conditions was performed at room temperature. Generally, the experimental intensity $v s$ time signal produced in a solid sample by the on resonance excitation of ${ }^{1} \mathrm{H}$ nuclei can be reproduced through a linear combination of different analytical functions by means of a suitable nonlinear leastsquares fitting procedure. Each function corresponds to a different dynamic domain of the sample [20]. The bestfitting parameters of the function are related to the dynamic behaviour of the domain, while the percent weight of the function is proportional to the number of protons present in the domain. In all cases the FID was well reproduced by a linear combination of Gaussian, Weibullian and exponential functions, having the general expression $f(t)=e^{-\left(t / T_{2}\right)^{n}}$. Here, $n$ is equal to 1,2 , and to a number between 1 and 2, for exponential, Gaussian and Weibullian functions, respectively; $T_{2}$ is the spin-spin relaxation time, which increases with increasing molecular mobility. The results of the FID analysis of CMCh, TMC and MCPS samples are reported in Table 2. Both TMC and CMCh are characterized by a very rigid fraction, described by a Gaussian function with a weight of $58-59 \%$ and a $T_{2}$ of less than $20 \mu \mathrm{s}$; the remaining fraction is represented by an exponential function with a $T_{2}$ of $300 \mu \mathrm{s}$ for $\mathrm{CMCh}$, and by a $2^{\text {nd }}$ Gaussian function $\left(T_{2} \approx 60\right.$ $\mu \mathrm{s})$ together with a very slowly decaying Weibullian function ( $T_{2}$ of the order of $\mathrm{ms}$ ) for TMC. Therefore, the rigid fraction of both these samples, reasonably associated with protons on the polymeric main chains, shows similar dynamic behaviour; on the contrary, the most mobile fraction, essentially identified with the side chains, experiences much faster dynamics for TMC, in agreement with the presence of a trimethylammonium group for which the reorientational motions should take place with a very low energetic barrier. Like for CMCh, the FID of the MCPS sample can be well reproduced by a linear combination of a Gaussian and an exponential function. However, now both these functions are characterized by shorter $T_{2}$ values, indicating a general decrease of mobility. This change is particularly important in the mobile fraction, where $T_{2}$ decreases by a factor of 2 with respect to $\mathrm{CMCh}$ and by one order of magnitude with respect to TMC. This is in agreement with the most significant changes observed in the ${ }^{1} \mathrm{H}$ MAS spectrum of MCPS with respect to the pure polyions, showing the broadening and displacement of the sole peaks corresponding to the $\mathrm{CH}_{2}$ group of $\mathrm{CMCh}$ and $\mathrm{CH}_{3}$ group of TMC, both belonging to side-groups, confirming the high-resolution ${ }^{13} \mathrm{C}$ results. These are clear indications of the presence, in the MCPS, of interactions between the most mobile moieties of CMCh $(\mathrm{N}$ carboxymehtyl) and TMC (trimethylammonium). Furthermore, it must be noted that the lyophilized MCPS sample subjected to compression to give a tablet showed the same ${ }^{1} \mathrm{H}$ FID behaviour as the non-compressed sample (results not shown), thus indicating that sample compression causes no changes of dynamic properties at a molecular level.

\section{Drug Release from PEC MCPS}

For studying drug release from MCPS, the gastric environment was avoided because the PEC would dissociate and dissolve at acidic $\mathrm{pH}$. At this $\mathrm{pH}$, indeed, the carboxyls of $\mathrm{CMCh}$ become non-ionized, the interpolymer interactions weaken dramatically, and both component polymers of the PEC are soluble. Hence, release was studied at pH 7.4 (SIF). The lyophilized MCPS would readily hydrate and disperse in SIF. However, the release of either FD4 or DMS was complete in $2 \mathrm{~h}$, a term inadequate for a sustained-release system. Such a comparatively rapid release is ascribed to a high porosity of the PEC hydrogel and a high specific surface area 
Table 2. Best Fitting Parameters Obtained from the 1H FID Analysis of CMCh, TMC and Lyophilized MCPS Samples Using a Linear Combination of Functions

\begin{tabular}{|c|c|c|c|c|c|c|c|c|c|}
\hline \multirow{3}{*}{ Sample } & \multicolumn{9}{|c|}{ Function } \\
\hline & \multicolumn{2}{|c|}{ Gaussian } & \multicolumn{2}{|c|}{ Gaussian } & \multicolumn{3}{|c|}{ Weibullian } & \multicolumn{2}{|c|}{ Exponential } \\
\hline & $\mathrm{w}, \%$ & $\mathbf{T}_{2}, \mu \mathrm{s}$ & $\mathrm{w}, \%$ & $\mathbf{T}_{2}, \mu \mathrm{s}$ & $\mathbf{n}$ & $\mathbf{w}, \%$ & $\mathbf{T}_{2}, \mathbf{m s}$ & $\mathrm{w}, \%$ & $\mathbf{T}_{2,} \boldsymbol{\mu \mathrm { s }}$ \\
\hline TMC & 57.7 & 19.4 & 14.1 & 61.6 & 1.36 & 28.2 & 1.87 & - & - \\
\hline $\mathrm{CMCh}$ & 59.3 & 16.6 & - & - & - & - & - & 40.7 & 300 \\
\hline MCPS & 70.7 & 13.9 & - & - & - & - & - & 29.3 & 135.4 \\
\hline
\end{tabular}

of the MCPS. In order to drastically reduce the latter, MCPS were compressed into tablets, the swelling and drug release properties of which were investigated.

\section{Swelling and Release Properties of Tablet Matrices Based on PEC MCPS}

The swelling profiles of tablet matrices obtained from PEC MCPS, loaded with FD4 or DMS, or drug free (control), are shown in Fig. (4), while the relevant release data are presented in Fig. (5). As can be verified, the swelling ratio in all cases attained $80 \%$ of its maximum value in less than $1 \mathrm{~h}$, whereas the dose fraction released in an hour's time was always about $20 \%$. Hence, drug release was not governed by matrix swelling. Probably, the swollen hydrogel acted as a diffusional barrier influencing the release kinetics. The profiles in Fig. (4) show that the matrices loaded with either FD4 or DMS absorbed water to increase their weights about 7-fold in 2-3 h. These findings confirm the high porosity of the PEC walls of the hydrated MCPS that was deduced from release data in the preceding section.

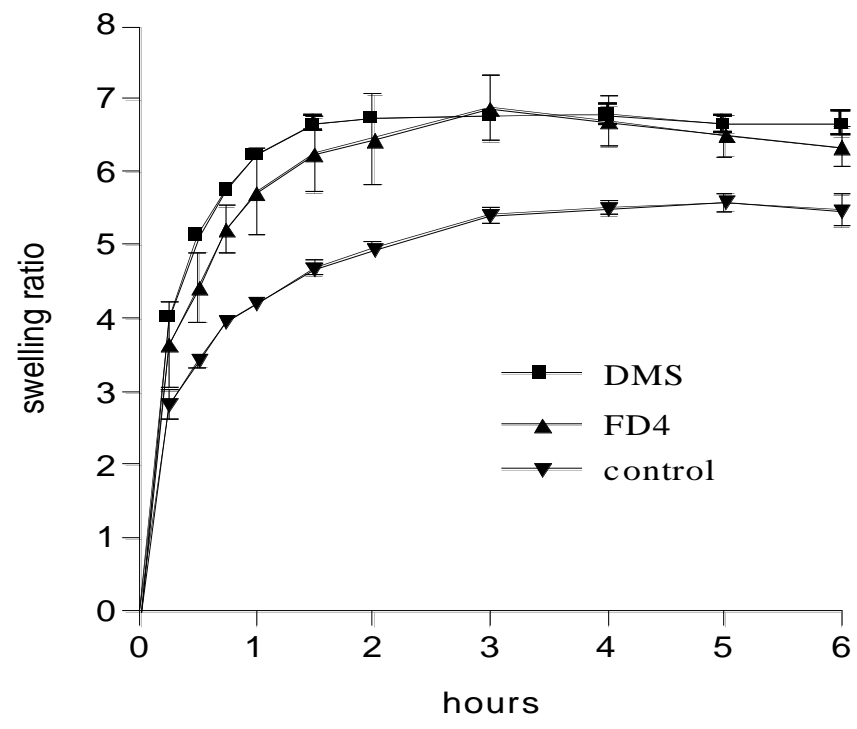

Fig. (4). Swelling (ratio of swollen to initial dry weights) profiles in water of DMS, FD4 and drug-free (control) PEC-based matrices obtained by MCPS compression. Data points are the means \pm SD of at least three values.

The swelling of matrices obtained from drug-free MCPS was significantly lower, which might be related to a denser packing of the drug-free MCPS lyophilizate. Although the swelling kinetics were measured over $6 \mathrm{~h}$, nevertheless the matrices were kept in water at $37^{\circ} \mathrm{C}$ for $24 \mathrm{~h}$. Over this pe- riod no sign of matrix disintegration was observed. Also, the matrices retained their original shape despite the swelling.

In order to evaluate the potential of tablets as drug delivery systems, their transit through ileum and ascending colon was simulated. When the ileum $\mathrm{pH}$ of 7.4 was changed into the $\mathrm{pH}$ of the ascending colon (5.5-6), a gradual disintegration at the matrix surface with liberation of the original MCPS was observed. On the other hand, when the whole release experiment was run with phosphate buffer $\mathrm{pH} 7.4$ as the dissolution medium no apparent disintegration occurred.

This is considered both a consequence and a sign of the $\mathrm{pH}$-sensitiveness of the PEC. In order to ascertain whether the salts of the buffer would play a specific role, either an acetate or a phosphate $\mathrm{pH} 6$ buffer was used to simulate the ascending colon environment: no apparent difference in the disintegration phenomenon was noticed. A visual comparison of release data in Fig. (5), obtained with different elution patterns, suggests that such a phenomenon produced a significant effect on the release kinetics of either DMS or FD4 and that the effect was independent of the particular pH-6 buffer. For a statistical treatment, Eq. (1) was fitted to the release data obtained with the different elution patterns and the resulting parameters were compared on the basis of the respective confidence intervals. The fitting is good in all cases, as appears from the data points and respective calculated curves in Fig. (5) up to 50-60\% release, and from the relevant $r^{2}$ values in Table 3. A survey of this table shows that in neither the DMS nor the FD4 case is the rate parameter, $K$, significantly different for the different elution patterns. As for the time exponent, $n$, in both of the DMS and FD4 cases the values of this parameter for the $\operatorname{SIF}(2 \mathrm{~h})$ $\mathrm{SACE} / \mathrm{PB}(4 \mathrm{~h})$ and $\operatorname{SIF}(2 \mathrm{~h})-\mathrm{SACE} / \mathrm{AB}(4 \mathrm{~h})$ patterns are not significantly different from each other whereas they are significantly higher than the value for the $\operatorname{SIF}(6 \mathrm{~h})$ pattern. This analysis confirms matrix disintegration being $\mathrm{pH}$-dependent, and quantifies the effect of this phenomenon to reduce the curvature of release profile and the decline of release rate. A similar behaviour is predicted of matrices administered in vivo to the ileum. A further release experiment was aimed at verifying whether the enzymatic hydrolysis of glycoside bonds in the chitosan backbone, known to occur in the ascending colon [21-23], would exert some effect on the release profile. To this purpose the release from the FD4 matrix was measured by applying the elution pattern $\operatorname{SIF}(2 \mathrm{~h})$ $\mathrm{SACE} / \mathrm{PB} / \mathrm{CC}(4 \mathrm{~h})$. Rat cecal microflora was used because of the similarity with human intestinal microflora [24]. FD4 was chosen because with this model drug a volume of $\mathrm{SACE} / \mathrm{PB} / \mathrm{CC}$ sufficient to ensure sink conditions during the release experiment could be prepared using the cecal con- 
Table 3. Parameters Obtained from the Fitting of Eq. (1) to the Release Data of Fig. (5)

\begin{tabular}{|c|c|c|c|c|}
\hline Drug & Elution Pattern & $\boldsymbol{K} \pm \mathbf{S E}(\mathbf{C I})$ & $\boldsymbol{n} \pm \mathbf{S E}\left(\mathbf{C I}^{\mathrm{a}}\right)$ & $\boldsymbol{r}^{\mathbf{2}}$ \\
\hline \hline DMS & $\operatorname{SIF}(2 \mathrm{~h})-\mathrm{SACE} / \mathrm{PB}(4 \mathrm{~h})$ & $20.8 \pm 0.3(20.1$ to 21.5$)$ & $0.60 \pm 0.01(0.58$ to 0.62$)$ & 0.9982 \\
\hline & $\operatorname{SIF}(2 \mathrm{~h})-\mathrm{SACE} / \mathrm{AB}(4 \mathrm{~h})$ & $20.0 \pm 0.5(19.0$ to 21.1$)$ & $0.60 \pm 0.02(0.56$ to 0.63$)$ & 0.9954 \\
\hline & $\operatorname{SIF}(6 \mathrm{~h})$ & $19.3 \pm 0.40(18.4$ to 20.2$)$ & $0.48 \pm 0.01(0.45$ to 0.51$)$ & 0.9940 \\
\hline FD4 & $\operatorname{SIF}(2 \mathrm{~h})-\mathrm{SACE} / \mathrm{PB}(4 \mathrm{~h})$ & $21.7 \pm 0.41(20.7$ to 22.7$)$ & $0.72 \pm 0.02(0.68$ to 0.76$)$ & 0.9979 \\
\hline & $\operatorname{SIF}(2 \mathrm{~h})-\mathrm{SACE} / \mathrm{AB}(4 \mathrm{~h})$ & $21.7 \pm 0.75(19.8$ to 23.5$)$ & $0.74 \pm 0.03(0.66$ to 0.81$)$ & 0.9935 \\
\hline & $\operatorname{SIF}(6 \mathrm{~h})$ & $21.2 \pm 0.71(19.5$ to 23.0$)$ & $0.59 \pm 0.03(0.51$ to 0.66$)$ & 0.9904 \\
\hline
\end{tabular}

tents from a single rat. The release profile obtained by this elution pattern (not reported) was superimposable to that obtained in the absence of enzymes, i.e., with the $\operatorname{SIF}(2 \mathrm{~h})$ $\mathrm{SACE} / \mathrm{PB}(4 \mathrm{~h})$ pattern, reported in Fig. (5b). Since no enzyme effects on release kinetics could be evidenced, it can be concluded that the enzyme degradation of the chitosan backbone is slower than FD4 release from matrix.

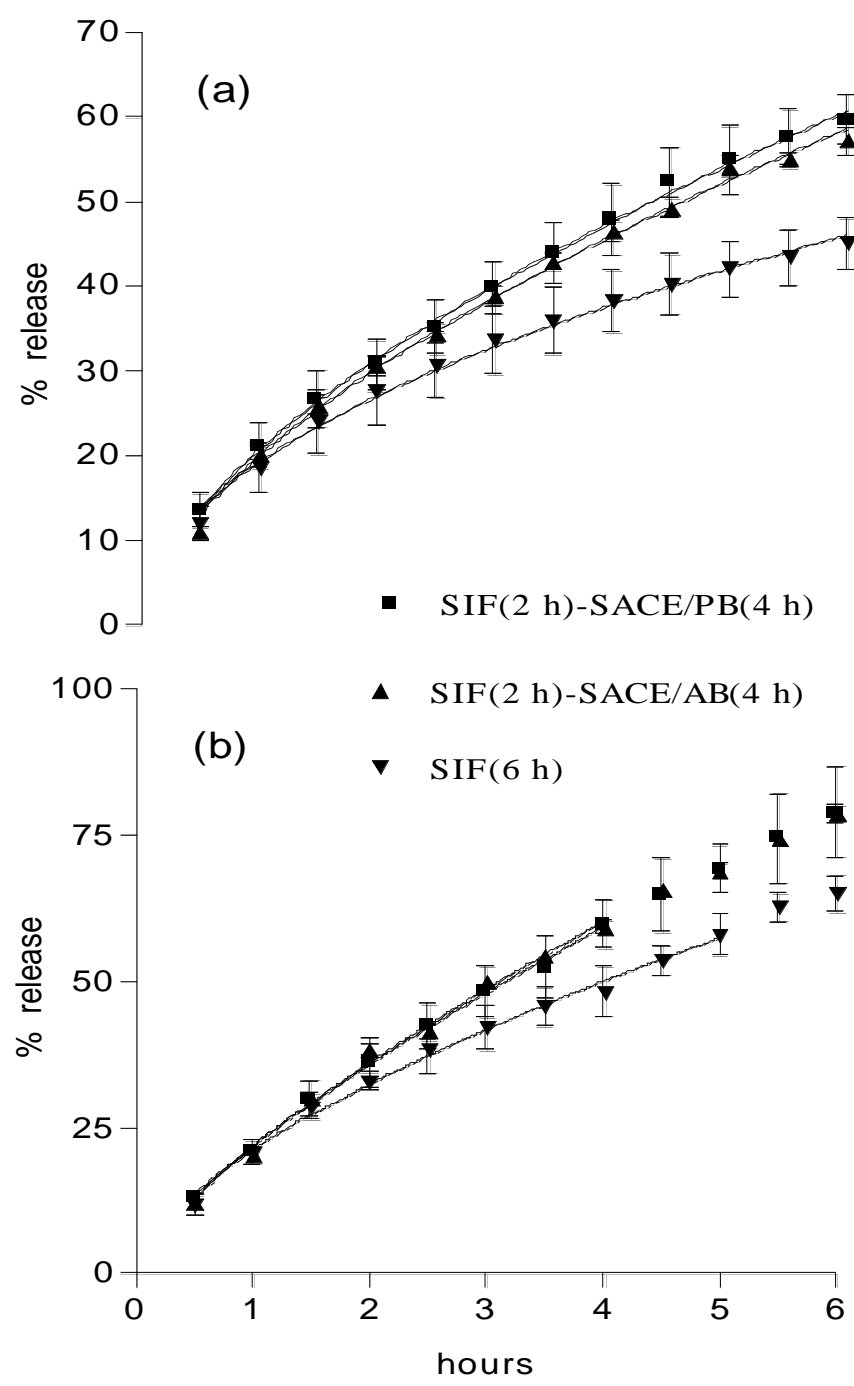

Fig. (5). Release profiles obtained by applying different elution patterns to DMS (a) or FD4 (b) PEC-based matrices obtained by MCPS compression. Data points are the means \pm SD of at least four values. Lines show the fitting of Eq. (1) to release data.

\section{Realizing Controlled Drug Release to Proximal Colon}

The PEC-based matrices were designed to start drug release in the distal small intestine and complete it in the proximal colon.

To realize this purpose the drug delivery system should prevent drug release in the stomach and the proximal small intestine. It was shown that hydroxypropylmethylcellulose capsules, enteric-coated with Eudragit ${ }^{\circledR}$ FS 30 D by an industrially viable process, disintegrated towards the distal small intestine and proximal colon [25]. Capsules of this type, of size 00 , could be used to carry the present matrices through the upper GI tract as far as the ileum, where they would be liberated intact, ready to start drug release. Alternatively, the matrices could be enteric-coated directly. The latter process was actually attempted in the present work, using Eudragit ${ }^{\circledR}$ $\mathrm{S} 100$ as the film-forming polymer, insoluble at $\mathrm{pH}<7$, and methanol as the solvent. The release from enteric-coated DMS PEC-based matrices was measured in vitro, applying a $\operatorname{SGF}(2 \mathrm{~h})-\operatorname{SJF}(2 \mathrm{~h})-\mathrm{SIF}(2 \mathrm{~h})-\mathrm{SACE} / \mathrm{PB}(4 \mathrm{~h})$ elution pattern, intended to simulate matrix transit across stomach, proximal small intestine, distal small intestine, and proximal colon. The resulting release profile, presented in Fig. (6) shows an initial burst, ascribed to the release of some DMS having diffused from matrix into the Eudragit film during the coating process, followed by a stop of release at the jejunum $\mathrm{pH}$ of 6.8 .

As soon as the protective film dissolved, upon attainment of the ileum $\mathrm{pH}$ of 7.4, drug release took place with a pattern corresponding to that of the uncoated matrix shown in Fig (5a, squares).

The data demonstrate that the release was effectively hindered by the enteric film during matrix transit across stomach and proximal small intestine, and that the coating process induced no modification of the release properties of matrix in the distal small intestine and proximal colon.

\section{CONCLUSIONS}

The oppositely charged polyelectrolyte chitosan derivatives CMCh and TMC have shown the ability to form a PEC at neutral $\mathrm{pH}$. PEC MCPS have been shown to be formed by virtue of the comparatively high viscosity of CMCh. Such MCPS have entrapped either a solution of a macromolecular drug model or a microparticulate dispersion of a sparingly soluble drug. MCPS are not, as such, able to adequately sustain drug release, because of a high specific surface and a 


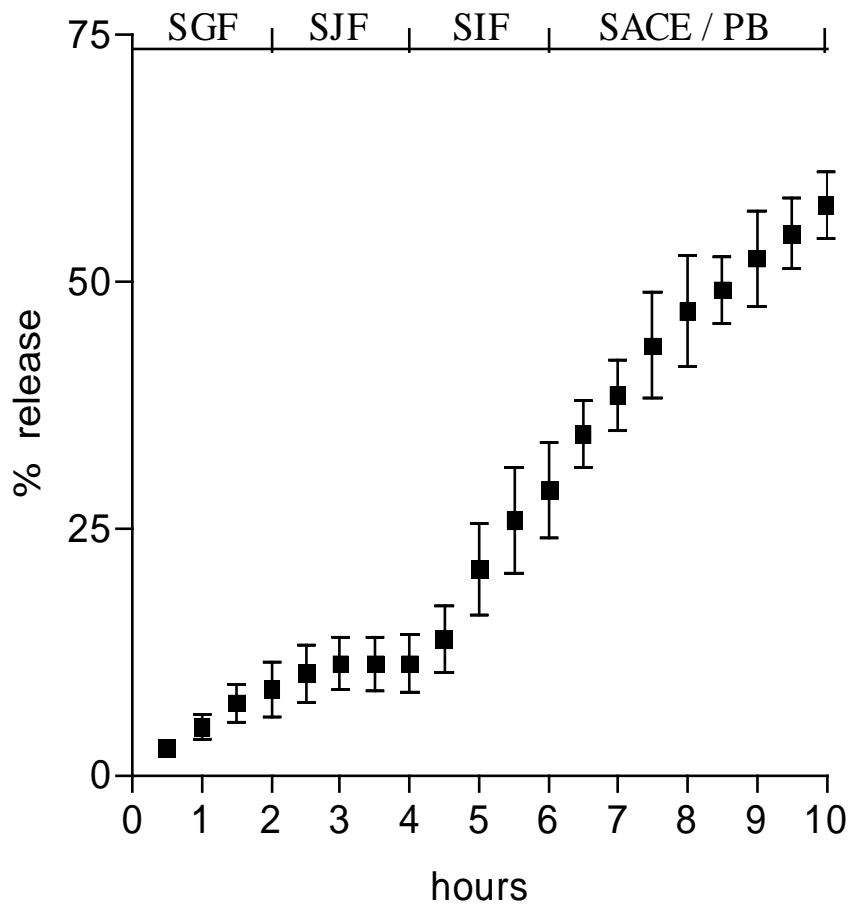

Fig. (6). Data on DMS release from enteric-coated PEC-based matrices obtained by MCPS compression. Elution pattern, $\operatorname{SGF}(2 \mathrm{~h})$ $\operatorname{SJF}(2 h)-\operatorname{SIF}(2 h)-S A C E / P B(4 h)$. Data points are the means \pm SD of at least four values.

high porosity of the hydrated PEC, yet they can readily be lyophilized and compressed into tablets which, once enteric coated with Eudragit S100, represent potential devices for the controlled and sustained delivery of macromolecular or sparingly soluble drugs to the distal small intestine and proximal colon. It has been demonstrated by solid-state NMR that the formation of the PEC between CMCh and TMC is due to electrostatic interactions between the carboxylate groups of $\mathrm{CMCh}$ and the quaternary ammonium groups of TMC. Because the former are $\mathrm{pH}$-sensitive, the PEC itself is $\mathrm{pH}$-sensitive. In fact, the change of the environmental $\mathrm{pH}$ from ileum to proximal colon caused some liberation of MCPS from the matrix surface, which contributed to sustaining the release rate. The present PEC MCPS have the essential advantage of providing a means of uniformly loading controlled doses of macromolecular or microparticulate drugs into a physically crosslinked hydrogel matrix. Indeed, the drug load could in principle be readily modulated by varying the drug concentration in the $\mathrm{CMCh}$ solution used to prepare the MCPS. An important issue with the present MCPS is the entrapment efficiency, which has proved very low with the model macromolecular drug. This issue is supposed to be addressed when dealing with a real drug, the encapsulation efficiency of which would have to be optimized. It should be considered in this respect that polypeptide drugs might show an interactivity with the PEC much stronger than that showed by FD4. Another problem, i.e., the poor flow properties of the lyophilized MCPS, will have to be addressed when transferring the MCPS compression process from laboratory to industrial scale.

\section{ACKNOWLEDGEMENTS}

The present investigation was supported by the Italian Ministry of University and Research (MIUR, PRIN 2005 035525). The Italian "Fondazione Cassa di Risparmio di Pisa" is thanked for partial financial support. Dr. Piero Narducci is thanked for providing SEM micrographs. Rofarma Italia is thanked for donating Eudragit S100 and triethyl citrate. Giusto Faravelli is thanked for donating ChitoClear FG90.

\section{REFERENCES}

[1] Peppas, N.A. In Preface Hydrogels in medicine and pharmacy, Peppas, N.A. Ed.; Fundamentals. CRC Press, 1986, Vol. 1.

[2] Hoffman, A.S. Adv. Drug Deliv. Rev., 2002, 54, 3.

[3] Berger, J.; Reist, M.; Mayer, J.M.; Felt, O. Eur. J. Pharm. Biopharm., 2004, 57, 35.

[4] Muzzarelli, R. Chitosan, In Natural Chelating Polymers, Muzzarelli, R. Ed., Pergamon Press: Oxford, 1973, pp. 144-176.

[5] Di Colo, G.; Zambito, Y.; Zaino, C. J. Pharm. Sci., 2007, in press.

[6] Muzzarelli, R.; Tanfani, F.; Emmanueli, M.; Mariotti, S. Carbohydr. Res., 1982, 107, 199.

[7] Di Colo, G.; Burgalassi, S.; Zambito, Y.; Monti, D.; Chetoni, P. J. Pharm. Sci., 2004, 93, 2851.

[8] Di Colo, G.; Zambito, Y.; Burgalassi, S.; Nardini, I.; Saettone, M.F. Int. J. Pharm., 2004, 273, 37.

[9] Dilova, V.; Zlatarova, V.; Spirova, N.; Filcheva, K.; Pavlova, A.; Grigorova, P. Boll. Chim. Farm., 2004, 143, 20.

[10] Cory, D.G.; Ritchey, W.M. J. Magn. Reson., 1988, 80, 128.

[11] Sandri, G.; Rossi, S.; Bonferoni, M.C.; Ferrari, F.; Zambito, Y.; Di Colo, G.; Caramella, C. Int. J. Pharm., 2005, 297, 146.

[12] Zambito, Y.; Zaino, C.; Di Colo, G. Eur. J. Pharm. Biopharm., 2006, 64, 16.

[13] Ritger, P.L.; Peppas, N.A. J. Control. Release, 1987, 5, 23.

[14] Saito, H. Magn. Reson. Chem., 1986, 24, 835.

[15] Saito, H.; Tabeta, R.; Hirano, S. Chem. Lett., 1981, 1479.

[16] Di Colo, G.; Baggiani, A.; Zambito, Y.; Mollica, G.; Geppi, M.; Serafini, M.F. Int. J. Pharm., 2006, 310, 154.

[17] Saito, H.; Tabeta, R.; Hirano, S. In Chitin Chitosan, Proceedings of the Second International Conference on Chitin and Chitosan, Hirano, S.; Tokura, S., Eds.; Japanese Society of Chitin and Chitosan, 1982: pp. 71-76.

[18] Calucci, L.; Galleschi, L.; Geppi, M.; Mollica, G. Biomacromolecules, 2004, 5, 1536.

[19] Borsacchi, S.; Cappellozza, S.; Catalano, D.; Geppi, M.; Ierardi, V. Biomacromolecules, 2006, 7, 1266.

[20] Hansen, E.W.; Kristiansen, P.E.; Pedersen, B. J. Phys. Chem., 1998, 102, 5444.

[21] Tozaki, H.; Komoike, J.; Tada, C.; Maruyama, T.; Terabe, A.; Suzuki, T.; Yamamoto, A. J. Pharm. Sci., 1997, 86, 1016.

[22] Park, H.S.; Lee, J.Y.; Cho, S.H.; Baek, H.J.; Lee, S.J. Arch. Pharm. Res., 2002, 25, 964.

[23] Zambito, Y.; Di Colo, G. J. Pharm. Pharm. Sci., 2003, 6, 274.

[24] Haberlin, B.; Friend, D.R. In Oral colon-specific drug delivery, Friend, D.R. Ed., CRC Press: Florida. 1992, 1-43.

[25] Cole, E.T.; Scott, R.A.; Connor, A.L.; Wilding, I.R.; Petereit, H.U.; Schminke, C.; Beckert, T.; Cadé, D. Int. J. Pharm., 2002, 231, 83. 